\title{
Criptococosis diseminada en anciano con VIH
}

\author{
Disseminated cryptococcosis in an elderly patient \\ with HIV
}

\author{
Ana María Escobar, Ricardo Díaz, Alfredo Posada • \\ Manizales (Colombia)
}

\section{Resumen}

Se presenta un paciente masculino de 79 años con un cuadro clínico de síntomas constitucionales, asociado a pleuresía linfocítica, meningitis e hidrocefalia, confirmándose posteriormente criptococosis diseminada y VIH-SIDA, con complicaciones asociadas a la terapia con anfotericina B y desenlace fatal. La criptococosis es una de las infecciones más frecuentes del SNC en pacientes con VIH y se han identificado varios factores que confieren mal pronóstico. En infección por VIH, se considera paciente "anciano" a aquellos mayores de 50 años. En general las características clínicas son similares a los pacientes más jóvenes, pero los adultos mayores, especialmente después de 65 años de edad, tienen más probabilidad de desarrollar SIDA en uno a tres años, mayor riesgo de diseminación de infecciones oportunistas y mayor mortalidad. En Colombia para el año 2007, según registros del Ministerio de Protección Social, había 5283 personas con SIDA mayores de 45 años (Acta Med Colomb 2011; 36: 85-89).

Palabras clave: criptococosis pleural, criptococosis meníngea, VIH, SIDA en ancianos.

\begin{abstract}
We report the case of a 79-year-old male patient with a clinical picture of constitutional symptoms associated with lymphocytic pleurisy, meningitis, and hydrocephalus. Disseminated cryptococcosis and HIV/AIDS were later confirmed. Complications of therapy with amphotericin B arose and the patient died. Cryptococcosis is one of the most common CNS infections in patients with HIV. Several factors that indicate a poor prognosis have been identified. In HIV infection, the patient is considered "old" above 50 years of age. The clinical features of these patients are generally similar to those found in younger patients; however, especially after the age of 65 years, these patients are more prone to developing AIDS over a period of 1-3 years, and their risk of acquiring disseminated opportunistic infections is increased. Their mortality rates are also higher. In Colombia, during 2007, according to the records of the Ministry of Social Protection, there were 5283 people older than 45 years with AIDS (Acta Med Colomb 2011; 36: 85-89).
\end{abstract}

Keywords: pleural cryptococcosis, meningeal cryptococcosis, HIV, AIDS in the elderly.

\author{
Dra. Ana María Escobar: Residente de Me- \\ dicina Interna; Dr. Ricardo Díaz: Neurólogo \\ Clínico, Docente; Dr. Alfredo Posada: Inter- \\ nista Neumólogo, Docente. Universidad de \\ Caldas. Manizales (Colombia). \\ Correspondencia: Dr. Ricardo Díaz. Clínica \\ de la Presentación. Manizales, Colombia. \\ E-mail: ridiaz@une.net.co \\ ana8_maria@hotmail.com \\ (Dra. Ana María Escobar) \\ Recibido: 16/VI/2011 Aceptado: 14/IV/2011
}

\section{Introducción}

Cuando se analizan como grupo, las infecciones neurológicas ocurren con cierta frecuencia y deben considerarse en el diagnóstico diferencial de muchos síndromes neurológicos. La capacidad limitada del cerebro para recuperarse de una agresión de esta naturaleza, hace esencial un diagnóstico rápido, preciso y por tanto tener la opción de un tratamiento adecuado. Las neuroinfecciones del tipo meningitis se dividen en agudas y crónicas, siendo estas últimas las que han tenido un curso de cefalea, fotofobia, fiebre, rigidez de nuca y cambios en el LCR, igual o mayor a cuatro semanas. En este grupo se ubican las infecciones micóticas del SNC, siendo la criptococosis una de las principales representantes. De otro lado, la presentación clínica de una infección por VIH puede darse con manifestaciones sistémicas de enfermedades oportunistas diseminadas. A continuación describimos una criptococosis con compromiso pleural y neurológico en un paciente adulto mayor VIH positivo.

\section{Descripción del caso clínico}

Paciente masculino de 79 años, abogado, con cuadro clínico que inició a principios de junio de 2009 , con tos seca, pérdida de peso, disfagia intermitente, inapetencia y malestar general, sin fiebre. Tres semanas después, acude a consulta en la cual se detectan ruidos cardiacos arrítmicos y disminución del murmullo vesicular en hemitórax izquierdo. 
Una Rx de tórax revela derrame pleural izquierdo, se evalúa por neumología y se realiza toracentesis y biopsia pleural, con líquido compatible con exudado (relación proteínas pleurales/plasmáticas 0.58 , relación lactato deshidrogenasa (LDH) 0.65 , colesterol 79), leucocitos 250, células mesoteliales (-), tinciones Z-N (-), gram (-), $\mathrm{KOH}(-)$ y coloraciones especiales de PAS y Grocott que reportaron hallazgos compatibles con micosis profunda, sugestiva de blastomicosis o paracoccidioidomicosis, según informe de patología. Con el fin de precisar diagnóstico, dos semanas después se realiza nueva toracentesis para cultivo de hongos y test de adenosina deaminasa (ADA). Este líquido es compatible de nuevo con exudado linfocitario (leucocitos 1,440 con $93 \%$ de linfocitos), con cultivo para hongos (-) y test de ADA (+) 50.1U/L. Se envía pieza patológica pleural a revisión por patólogo neumólogo, quien confirma micosis y se tipifica como criptococosis (Figura 1). Se inicia entonces fluconazol $200 \mathrm{mg} / \mathrm{día}$, con lo cual se obtiene mejoría progresiva del paciente.

Luego de la mejoría lograda en síntomas respiratorios, apetito y peso, 15 días después el paciente desarrolla cefalea, cambios en esfera mental, trastorno de la marcha e incontinencia urinaria. Se ordena TAC cerebral que mostró hidrocefalia obstructiva comunicante simétrica, sin lesiones parenquimatosas. Se remite a neurología, que practica punción lumbar, con LCR que mostró 100 leucocitos (100\% linfocitos), proteínas $39.7 \mathrm{mg} / \mathrm{dL}$, glucorraquia 32 $\mathrm{mg} / \mathrm{dL}$ (relación de glucosa LCR/ sérica 0.18), con gram, $\mathrm{Z}-\mathrm{N}$, tinta china y cultivo negativos. Con este resultado se decide aumentar dosis de fluconazol a $400 \mathrm{mg} /$ día. Se le explica al paciente la necesidad de realizar examen para $\mathrm{VIH}$, pero rechaza enfáticamente este estudio. Negaba conductas sexuales de riesgo, farmacodependencia y refería no haber tenido relaciones sexuales con su esposa en los últimos 15 años.

La evolución durante los siguientes cuatro meses fue satisfactoria, hasta finales de diciembre, cuando presenta cuadro gripal con reaparición de astenia marcada, postración, inapetencia, cefalea y confusión mental. Al examen físico presentaba múltiples lesiones de dermatitis seborreica en cara y tronco, somnolencia, desorientación en tres esferas, rigidez nucal, sin compromiso de pares craneales ni papiledema y sin signos de focalización motora o sensitiva. Se realizó TAC cerebral que mostró persistencia de la hidrocefalia, sin lesiones expansivas y nuevo estudio de LCR reportado con células 0 , proteínas $108 \mathrm{mg} / \mathrm{dL}$, glucorraquia 19 (relación de glucosa LCR/sérica 0.24), gram (-), Z-N (-) y tinta china (+) para criptococo. Con estos resultados se insiste en la necesidad de realizar prueba para VIH, la cual es finalmente autorizada por la esposa, dadas las alteraciones en la esfera mental del paciente. La prueba para ELISA VIH es positiva al igual que la prueba de Western blot (cuatro bandas presentes: gp 120, gp 41, p 31 y p17).

Es valorado por el servicio de infectología que recomienda aumentar dosis de fluconazol a $800 \mathrm{mg} /$ día por vía

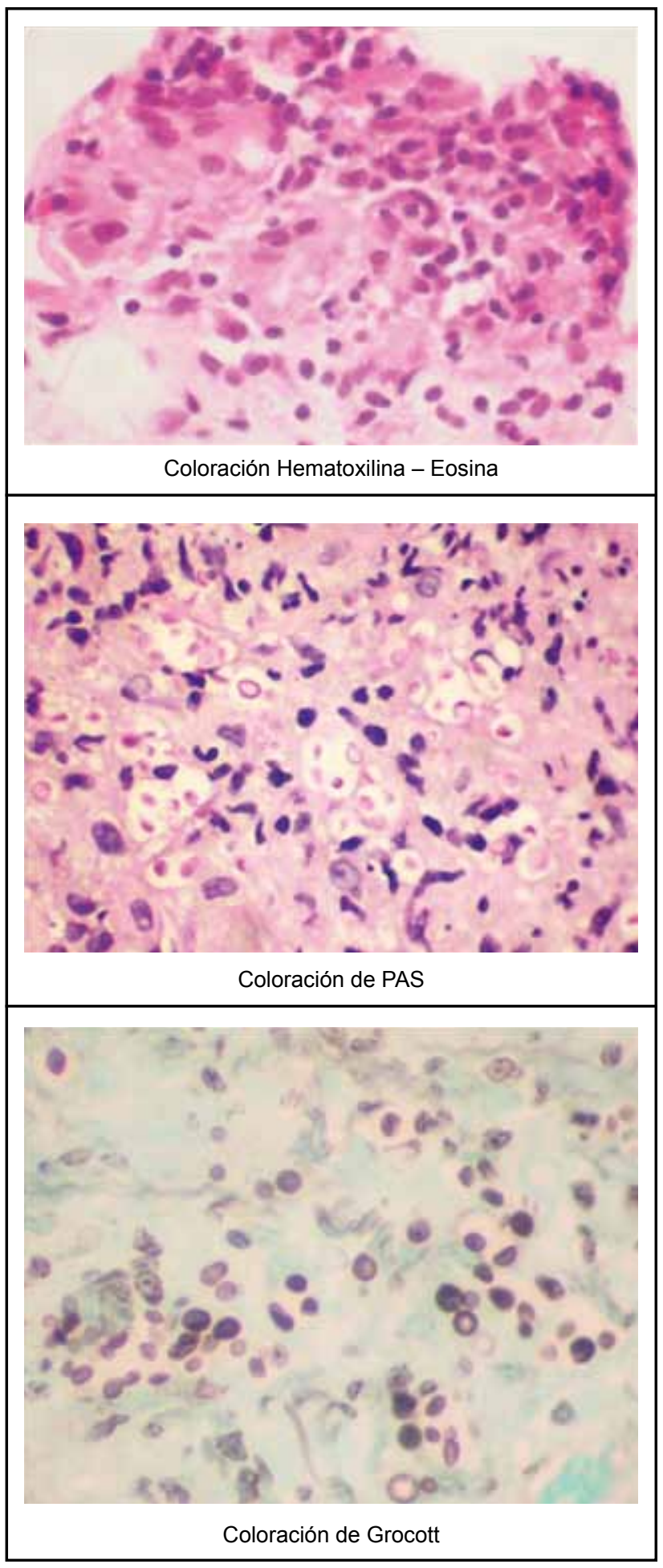

Figura 1. Coloraciones especiales en biopsia pleural.

intravenosa y no aconseja anfotericina B debido a valores de creatinina y tasa de filtración glomerular calculada (33 $\mathrm{ml} / \mathrm{min}$ ). Adicionalmente recomendó profilaxis con trimetroprim-sulfa y conveniencia de iniciar antirretrovirales.

Por razones administrativas de su seguridad social se traslada a otra institución de la ciudad donde es valorado por 
nuevo equipo médico. Allí se inicia anfotericina B IV $16 \mathrm{mg}$ $(0.25 \mathrm{mg} / \mathrm{kg})$, con aumento al $4^{\circ}$ día a $0.5 \mathrm{mg} / \mathrm{kg}$, momento en el que desarrolla fibrilación auricular con compromiso hemodinámico y edema pulmonar, que requiere traslado a UCI y soporte ventilatorio.

Se disminuye dosis de anfotericina a $0.25 \mathrm{mg} / \mathrm{kg}$, pero el paciente continúa en malas condiciones, con elevación progresiva de creatinina y potasio (creatinina desde 1.34 $\mathrm{mg} / \mathrm{dL}$ hasta $6.3 \mathrm{mg} / \mathrm{dL}$ y potasio desde $4.2 \mathrm{mEq} / \mathrm{L}$ hasta $6.9 \mathrm{mEq} / \mathrm{L}$ ), con evolución a coma profundo y muerte. La anfotericina B se administró durante 19 días (dosis total 326 $\mathrm{mg}$ ) hasta el día previo a su fallecimiento.

\section{Discusión}

La presentación clínica de este caso se dio con síntomas constitucionales y manifestaciones respiratorias con derrame pleural de tipo exudado linfocítico. Dentro de las causas de esta variedad de derrame pleural se incluyen neoplasias, tuberculosis, micosis, pleuresía reumatoidea, lupus eritematoso sistémico, sarcoidosis y síndrome de uñas amarillas (1), si bien más de $90 \%$ de las etiologías corresponden a tumores e infecciones (2).

Las neoplasias asociadas a derrame pleural más frecuentes son carcinoma broncogénico, carcinoma de mama, linfoma, mesotelioma, sarcoma y melanoma (3). Un examen eficiente y mínimamente invasivo es la citología de líquido pleural, la cual logra establecer el diagnóstico en la mayoría de los casos (4). Su sensibilidad oscila entre $40 \%$ y $87 \%$ para neoplasias en general, siendo $70 \%$ para el adenocarcinoma metastásico. Si se toman más muestras citológicas, el rendimiento de la prueba puede aumentar $30 \%$ (2). Pruebas adicionales en el líquido pleural son la determinación de $\mathrm{LDH}$, que en cáncer es usualmente mayor de $320 \mathrm{U} / \mathrm{L}$ (4) y el test de ADA que en neoplasias suele ser menor de 38 U/L (5). La biopsia pleural puede establecer el diagnóstico de neoplasia en 45 a $60 \%$ de casos (3), obteniendo mayor rendimiento diagnóstico si se realiza por toracoscopia.

El siguiente diagnóstico diferencial más importante, especialmente en nuestro medio, es la tuberculosis. En el paciente, el test de Z-N y cultivo para micobacterias fueron negativos, sin embargo, el test de ADA fue positivo (50.1 $\mathrm{U} / \mathrm{L})$. Este último ha sido informado con una sensibilidad de 92\% para TBC (6), aunque su especificidad disminuye pues se asocia a otras entidades, como neoplasias, infecciones bacterianas (empiema), artritis reumatoidea y LES (7). Por lo anterior, se recomienda considerar niveles mayores de 40 $\mathrm{U} / \mathrm{L}$ como sugestivos de TBC, si bien por encima de $70 \mathrm{U}$ alcanzaría una mayor especificidad.

Las micosis son responsables únicamente del $1 \%$ de los derrames pleurales y las más asociadas a esta complicación son blastomicosis, paracoccidiodomicosis, histoplasmosis, coccidiodomicosis y criptococosis (8).

Blastomicosis y paracoccidiodomicosis se consideraron dentro de las micosis profundas en la primera revisión efectuada por patología; sin embargo, generalmente se comportan como neumonías rápidamente progresivas $(9$, 10). La blastomicosis no es usual en nuestro medio, en tanto que la paracoccidiodomicosis es endémica en Brasil, Venezuela y Colombia. En esta entidad la mayoría de pacientes desarrollan enfermedad diseminada a riñones, tracto genital y meninges con adenopatías cervicales prominentes y hepatoesplenomegalia $(9,11)$.

En el paciente se confirmó el diagnóstico de criptococosis mediante biopsia pleural no dirigida, situación poco frecuente en la práctica clínica. Al efectuar una revisión de la literatura sólo encontramos dos casos reportados en los que se identificó dicha infección mediante esta técnica diagnóstica $(14,15)$. Esta micosis se manifiesta con tos no productiva, pérdida de peso, cefalea, malestar general, dolor torácico y fiebre. Los hallazgos radiológicos son variados, entre ellos, nódulos, consolidaciones, opacidades en vidrio esmerilado, infiltrados reticulares, adenopatías y derrame pleural, siendo este último uno de los más frecuentes $(12,13)$.

Debe resaltarse que el diagnóstico de criptococosis pleural ya implica criptococosis diseminada, siendo los sitios más comunes de diseminación en piel, articulaciones, próstata, ojos y SNC. Una recomendación reciente en el manejo de pacientes con criptococosis pulmonar con riesgo de diseminación, como son personas mayores de 65 años, presencia de derrame pleural o ser VIH positivo, es la realización de punción lumbar, aun en ausencia de síntomas neurológicos (16), pues la diseminación a SNC ocurre en 65 a 94\% de los pacientes (17).

La incidencia de criptococosis en pacientes con SIDA varía entre 2 y $11 \%$, pudiendo ser la primera manifestación de VIH y presentarse concomitante con otras infecciones oportunistas (18). El síntoma principal es la cefalea, frecuentemente asociada con síntomas como vómito, fiebre, alteraciones del estado mental y signos meníngeos. El LCR de estos pacientes generalmente muestra pleocitosis linfocitaria, hiperproteinorraquia e hipoglucorraquia. Sin embargo, en cerca de $50 \%$ de los casos, algunos de estos parámetros del LCR pueden ser normales. La tinta china es positiva en $70 \%$ de individuos, mientras que el antígeno para criptococo en LCR es positivo en más de $90 \%$ (18). Para fines diagnósticos resulta de gran utilidad realizar cultivo y látex para antígeno de criptococo en sangre (títulos mayores de 1:8 se consideran diagnóstico presuntivo). Las neuroimágenes pueden ser normales, pero con frecuencia revelan hidrocefalia y, en $10 \%$ de los casos, se pueden encontrar lesiones de tipo masa (criptococomas) (19).

La mortalidad es de $30 \%$ y se mencionan como factores de mal pronóstico los siguientes: compromiso del nivel de conciencia, tinta china positiva en LCR, títulos de antígeno para criptococo altos $(\geq 1: 1024)$, pobre respuesta inflamatoria en LCR (< de 20 células), edad mayor a 60 años, imágenes anormales e hipertensión endocraneana (16).

En los últimos años se ha identificado a la criptococosis del SNC como una de las complicaciones neurológicas más comúnmente asociadas al síndrome de reconstitución 
inmune y usualmente comienza luego de uno a tres meses de inicio de la terapia antirretroviral (20).

Con relación al tratamiento, aunque algunos estudios han encontrado una efectividad terapéutica similar entre anfotericina B y fluconazol para meningitis criptococóccica no complicada (21), en la actualidad se recomienda iniciar manejo con anfotericina B $(0.7 \mathrm{mg} / \mathrm{kg} / \mathrm{d}$ á) con o sin flucitosina (no disponible en nuestro medio) por dos semanas, seguida por fluconazol (400 mg/día) por ocho a diez semanas. Si al cabo de este tiempo el cultivo de LCR para criptococo es negativo, la dosis puede reducirse a $200 \mathrm{mg}$ al día, manteniéndola de manera indefinida, puesto que alrededor de $50 \%$ de los pacientes que no reciben profilaxis secundaria presentan recaída de la enfermedad. Otras publicaciones recientes extienden el tratamiento con fluconazol $200 \mathrm{mg} /$ día hasta que el conteo de CD4+ sea igual o mayor a 200 células $/ \mathrm{mm}^{3}$ como resultado de la terapia antirretroviral $(11,16)$.

Si se tienen en cuenta los mecanismos de acción de los antifúngicos, los azoles (fluconazol, itraconazol, etc.) disminuyen la concentración de ergosterol, que es precisamente el sitio de unión de la anfotericina B (166), lo que podría generar resistencia a la acción de la anfotericina B si aquellos se usan en primera instancia.

Cuando este abordaje terapéutico se analiza desde los aspectos de tolerancia y seguridad, cabe mencionar que los efectos adversos del fluconazol son significativamente menores cuando se comparan con los de la anfotericina B. El daño renal es la complicación más temida de este fármaco y se produce por disminución dosis-dependiente de la tasa de filtración glomerular, dada por efecto vasoconstrictor directo en arteriolas renales; además, genera pérdida de potasio, magnesio, bicarbonato, eritropoyetina, y acidosis metabólica (16). Es por esto, que en los últimos años se recomiendan preparaciones lipídicas de anfotericina (dispersión coloidal, liposomal o complejo lipídico) por ser menos nefrotóxicas $(11,12,16)$.

En nuestro paciente, por diversos motivos, entre ellos la renuencia a practicarse la prueba de VIH, el diagnóstico de SIDA se realizó siete meses después del inicio de las manifestaciones clínicas. El paciente presentaba disfagia fluctuante, posiblemente dada por candidiasis esofágica, pérdida de peso mayor de $10 \%$, aparición reciente de lesiones extensas de dermatitis seborreica, leucopenia con linfopenia y trombocitopenia (22), todo lo cual sumado a la identificación de critptococosis pleural, hacían altamente probable la presencia de SIDA, si bien, otras enfermedades podrían dar un cuadro similar.

En pacientes ancianos no es usual incluir el SIDA dentro de las posibilidades diagnósticas. En Estados Unidos en el 2005, se calculó que las personas con SIDA, de 65 años o más, sobrepasaban los 10000 habitantes (23). En Colombia no disponemos de datos similares, pero en el año 2007, los mayores de 45 años con diagnóstico de SIDA correspondían a $13.1 \%$ de un total de 40335 registros, lo cual equivale a 5283 personas (24).
Un factor epidemiológico que adquiere importancia para este riesgo, es que de los hombres de 50 a 75 años sexualmente activos, cerca de $92 \%$ nunca usa preservativo masculino y hasta $96 \%$ de hombres y mujeres en estas edades nunca se han realizado pruebas de tamizaje para VIH (23).

En general, las características clínicas de VIH en ancianos son similares a las de pacientes más jóvenes, pero usualmente hay menos fiebre, nauseas, escalofríos, diarrea o lesiones en cavidad oral y, en cambio, presentan con más frecuencia pérdida de peso y síntomas neurológicos. Los pacientes mayores de 65 años tienen mayor probabilidad de desarrollar SIDA en un plazo de uno a tres años, y en general, presentan mayor mortalidad dada entre otros factores por infecciones oportunistas con mayor riesgo de diseminación (23).

Las indicaciones para iniciar terapia antirretroviral, son las mismas que en pacientes más jóvenes (23). En general, en pacientes con VIH y micosis sistémicas, se recomienda iniciar terapia antifúngica dos a diez semanas antes de la terapia antirretroviral con el fin de disminuir el riesgo del síndrome de reconstitución inmune $(11,16)$.

En conclusión, la infección VIH-SIDA se ha tornado en un problema de salud pública que puede afectar cualquier grupo etario y, como vemos en el presente caso, debemos considerar esta entidad en el diagnóstico diferencial de personas ancianas que se presenten con compromiso de su estado general, incluyendo pérdida de peso, muestren deterioro progresivo y que además desarrollen candidiasis, alteraciones hematológicas o neuroinfecciones. En el manejo de la criptococosis del SNC se recomienda iniciar tratamiento con anfotericina B intravenosa y posteriormente cambiar a fluconazol oral. Sin embargo, cuando esta situación clínica se presente en personas ancianas, podría optarse mejor por las preparaciones lipídicas de la anfotericina B dada su menor toxicidad renal y cardiaca como ocurrieron en el caso clínico descrito.

\section{Referencias}

1. Light R W. Pleural Effusion. N Engl J Med 2002; 346: 1971-7.

2. Dalbeth N, Lee G. Lymphocytes in pleural disease. Curr Opin Pulm Med 2005; 11: $334-9$

3. Villena V, Ferrer J, Hernández L, Gafas A de Po, Pérez E, Rodríguez F, et al. Diagnóstico y tratamiento del derrame pleural. Arch Bronconeumol 2006; 42: 349-72.

4. Burgess LJ. Biochemical analysis of pleural, peritoneal and pericardial effusions. Clinica Chimica Acta 2004; 343: 61-84.

5. Porcel JM, Alemán C, Bielsa S, Sarrapio J, Fernández T, Esquerda A. A decision tree for differentiating tuberculous from malignant pleural effusions. Respiratory Medicine 2008; 102: 1159-64.

6. Light R W. Pleural Diseases. 4th. ed. Philadelphia: Lippincott Williams \& Wilkins 2007.p.211-224.

7. Ernam D, Atalay F, Hasanoglu HC, Kaplan Ö. Role of biochemical tests in the diagnosis of exudative pleural effusions. Clinical Biochemistry 2005; 38: 19-23.

8. Restrepo A. Micosis Pulmonares. En: Chaparro C,Awad C, Torres C. Fundamentos de Medicina. Medellín-Colombia. CIB. 1998. 187-215.

9. Davies SF, Knox KS, Sarosi GA. Fungal Infections. Mason: Murray \& Nadel's Textbook of Respiratory Medicine, 5th ed. Philadelphia: Elsevier Saunders. 2005; Vol I: Chapter 36.

10. McKinnell JA, Pappas PG. Blastomycosis: New Insights into Diagnosis, Prevention, and Treatment. Clin Chest Med 2009; 30: 227-39. 
11. Sax PE, Cohen CJ, Kuritzkes DR. HIV Essentials. 3th. Ed. Boston, MA. Jones and Bartlett Publishers. 2010.p.94-6.

12. Huston SM, Mody CH. Cryptococosis: An Emerging Respiratory Mycosis. Clin Chest Med 2009; 30: 253-64.

13. Chang WCh, Tzao Ch, Hsu H, Lee S, Huang K, Tung H, et al. Pulmonary Cryptococcosis. Comparison of Clinical and Radiographic Characteristics in Immunocompetent and Immunocompromised Patients. Chest 2006; 129: 333-40.

14. Katz AS, Niesenbaum L, Mass B. Pleural effusion as the initial manifestation of disseminated cryptococcosis in acquired immune deficiency syndrome. Diagnosis by pleural biopsy. Chest 1989; 96: 440-1.

15. Thomas R, Cristopher DJ, Kurien S, Thangakunam B, James P, Gupta R. Cryptococcal pleural effusion in acquired immune deficiency syndrome-Diagnosis by closed pleural biopsy. Respiratory Medicine Extra 2006; 2: 7-9.

16. Rex JH, Stevens DA. Systemic Anifungal Agents. Mandell G L, Bennet J E, Dolin R. Mandell, Douglas, and Bennett's Principles and Practice of Infectious Diseases. $7^{\text {th }}$. Ed. Philadeplphia: Churchill Livingstone Elsevier. 2010; Vol I: Chapter 40

17. Clark R A, Greer D L, Valainis, G T, Hyslop N E. Cryptococcus neoformans pulmonary infection in HIV-1-infected patients. J Acquir Immune Defic Syndr 1990; 3: 480.

18. Berger J R, Espinosa P S. Complicaciones neurológicas del síndrome de inmu- nodeficiencia adquirida. En: Toro J, Yepes M, Palacios E. eds. Neurología. 2a ed. Bogotá: Manual Moderno; 2010.p.320-21.

19.Smith A, Smirnitopoulos J, Rushing E. Central Nervous System Infections Associated with Human Immunodeficiency Virus Infection: Radiologic-Patologic Correlation. RadioGraphics 2008, 28: 2033-58.

20. Shelburne SA 3rd, Darcourt J, White AC Jr, Greenberg SB, Hamill RJ, Atmar RL, et al: The role of immune reconstitution inflammatory syndrome in AIDSrelated Cryoticoccus neoformans disease in the era of highly active antiretroviral therapy. Clin Infect Dis 2005; 40:1049-52.

21. Powderly WG, Saag MS, Cloud GA, Robinson P, Meyer RD, Jacobson JM, et al. A controlled trial of fluconazole or amphotericin B to prevent relapse of cryptococcal meningitis in patients with the acquired immunodeficiency syndrome. N Engl J Med 1992; 326: 793-98.

22.Stone VE, Bounds BC, Muse VV, Ferry JA. Case 29-2009: An 81-Year-Old Man with Weight Loss, Odynophagia, and Failure to Thrive. N Engl J Med 2009; 361: 1189-98.

23. Luther V P, Wilkin A. HIV Infection in Older Adults. Clin Geriatr Med 2007; 23: $567-83$

24. Ministerio de la Protección Social. República de Colombia. Dirección General de Salud Pública. Grupo de Promoción y Prevención, 2007. 